\title{
The 17 min orbital period in the Ultra Compact X-ray Binary 4U 0513-40 in the X-ray energy band
}

\author{
M. Fiocchi* \\ INAF/IASF-Roma, Via Fosso del Cavaliere 100, I-00133, Roma, Italy \\ E-mail: mariateresa.fiocchi@iasf-roma.inaf.it;
}

A. Bazzano

INAF/IASF-Roma, Via Fosso del Cavaliere 100, I-00133, Roma, Italy

\section{Natalucci}

INAF/IASF-Roma, Via Fosso del Cavaliere 100, I-00133, Roma, Italy

\section{R. Landi}

INAF/IASF-Bologna, Via P. Gobetti 101, I-40129 Bologna, Italy

\section{P. Ubertini}

INAF/IASF-Roma, Via Fosso del Cavaliere 100, I-00133, Roma, Italy

\begin{abstract}
The principal motivations for studying the X-ray binaries is that the accretion onto neutron stars and black holes provides a unique window on the physics of the strong gravity and dense matter. For this reason, a renewed interest has developed in ultracompact X-ray binaries and their identification is very important, albeit complex because of the difficulty in measuring orbital periods in low mass X-ray binaries. The ultracompact low-mass X-ray binary 4U 0513-40 in the globular cluster NGC1851 reveals a clear sinusoidal periodic signal with a period of $\sim 17$ minutes when the source is in a typical high/soft state with a dominant soft thermal component (using BeppoSAX and Chandra data). The periodicity disappears when the source is in a low/hard state and the thermal soft component is no longer required any more to model the data (using XMM-Newton and INTEGRAL observations). These properties indicate the orbital nature of the detected signal and imply an high inclination angle of the binary system $\left(>80^{\circ}\right)$.
\end{abstract}

The Extreme and Variable High Energy Sky - extremesky2011,

September 19-23, 2011

Chia Laguna (Cagliari), Italy

${ }^{*}$ Speaker. 


\section{Introduction}

Ultracompact X-ray binaries (UCXBs) are systems with orbital periods $\left(P_{\text {orb }}\right)$ shorter than $\approx 1 \mathrm{hr}$ in which a neutron star or black hole accretes matter from a companion low mass star. UCXBs are rare objects and their identification is very difficult because of the difficulty to measuring $P_{\text {orb }}$ in LMXBs. The most recent compilation of ultracompact X-ray binaries lists 27 candidates (in 't Zand et al., 2007). Eight out of 52 LMXBs with measured orbital period are in the ultracompact regime (in 't Zand et al. 2007, Nelemans \& Jonker 2006). IBIS results from long-term monitoring of the UCXBs showed that these sources spend most of the time in the canonical low/hard state, with X-ray luminosities $\lesssim 7 \times 10^{36} \mathrm{erg} \mathrm{s}^{-1}$, plasma temperature $k T e \gtrsim 20 \mathrm{keV}$ and $\tau \lesssim 4-5$ (Fiocchi et al. 2008).

$4 \mathrm{U}$ 0513-40 is an X-ray binary in the Galactic globular cluster NGC 1851 with a 17-minute orbital modulation first observed with the Hubble Space Telescope (Zurek et al. 2009). It is a persistent source showing evidence for variability of a factor of $\sim 10$ in X-ray luminosity on timescales of $\sim$ weeks, and a factor of more than 20 overall (Maccarone et al. 2010).

\section{Observations and Data Analysis}

Table 1 gives a summary of source observations performed with instruments on board BeppoSAX, Chandra, XMM-Newton and INTEGRAL satellites.

The LECS, MECS and PDS/BeppoSAX (Boella et al. 1997) event files and spectra were generated with the Supervised Standard Science Analysis (Fiore, Guainazzi \& Grandi 1999). Both LECS and MECS spectra were accumulated in circular regions of 8 arcmin radius. The PDS spectra were extracted using the XAS version 2.1 package (Chiappetti \& Dal Fiume 1997). The Chandra (Weisskopf 1999) data were processed with the CIAO (Chandra Interactive Analysis of Observations) software, version 4.1.2, i.e. the same version of CALDB (Calibration Data Base), provided by the Chandra X-ray Center and following the science threads listed on the CIAO website ${ }^{1}$. We extracted source photons from a circular region centered on the source with an extraction region of 8 arcsec. For the background, we used circular source-free regions in the same CCD of the studied source. XMM-Newton (Turner et al. 2001) data have been processed starting from the observation files with SAS 7.0.0. X-ray events corresponding to patterns 0-4 were selected in the EPIC-pn camera. Source light curves and spectra were extracted from circular regions of $10 \operatorname{arcsec} c e n t e r e d ~ o n$ the source, while background products were obtained from off-set regions close to the source.

The analyzed INTEGRAL (Winkler et al. 2003) data consist of all public observations in which $4 \mathrm{U}$ 0513-40 was within the field of view of the IBIS and JEM-X detectors. Broad-band spectra, $\sim 5-80 \mathrm{keV}$, are obtained using data from the high-energy instruments, JEM-X (Lund et al. 2003) and IBIS (Ubertini et al. 2003). The IBIS and JEM-X data have been processed using the Off-line Scientific Analysis (OSA v. 9.0) software released by the INTEGRAL Science Data Center (ISDC, Courvoisier et al. 2003). These runs were performed with AVES cluster, designed to optimize performances and disk storage for the INTEGRAL data analysis (Federici et al. 2010).

\footnotetext{
${ }^{1}$ Available at http://cxc.harvard.edu/ciao/
} 
Table 1: Summary of the X-ray binary 4U0513-40 observations

\begin{tabular}{lcc}
\hline \hline Instrument & $\begin{array}{c}\text { Tstart } \\
\text { MJD }\end{array}$ & $\begin{array}{c}\text { Exposure } \\
\text { ks }\end{array}$ \\
\hline BeppoSAX/LECS & 51597.6 & 31.5 \\
BeppoSAX/MECS & 51597.6 & 73.6 \\
BeppoSAX/PDS & 51597.6 & 34.9 \\
XMM Newton/EPIC-pn & 52730.0 & 23.5 \\
CHANDRA/ACIS & 54560.6 & 18.8 \\
INTEGRAL/JEM-X & 53917.6 & 102.8 \\
INTEGRAL/IBIS & 53380.4 & 601.5 \\
\hline \hline
\end{tabular}
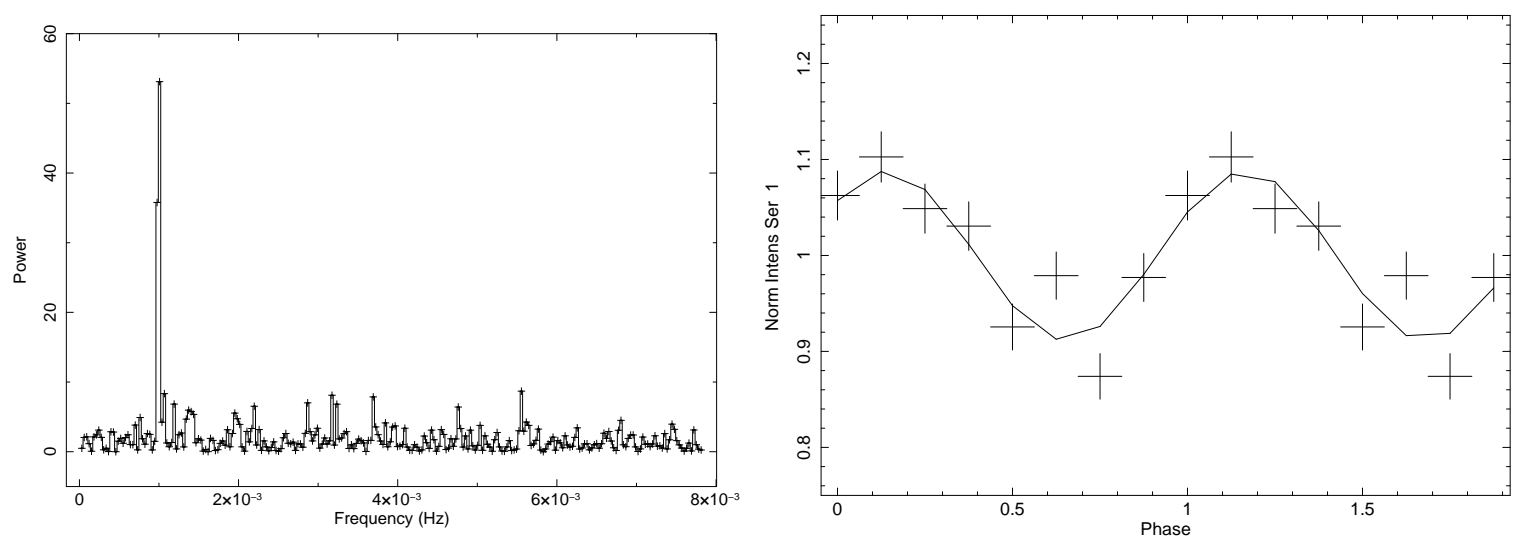

Figure 1: The power spectrum and efolding phase diagram using ACIS 0.3-5 keV data

\section{Temporal analysis}

Using the ACIS/Chandra data, we accumulated a 16s bin light curve in the 0.3-5 keV energy band of $4 \mathrm{U} 0513-40$ detected at $\sim 140 \sigma$ in this energy range and calculated a power spectrum over the whole observation following the method outlined by Israel \& Stella (1996). The power spectrum is shown in Figure 1 (left panel) where a peak at $0.00099 \mathrm{~Hz}$ is clearly observed. The 4U 0513-40 period was obtained with an epoch-folding technique. The best-fit period is (1004 \pm 18$)$ s with uncertainties at $1 \sigma$ confidence level. Using this period value, we folded the light curve and a sinusoidal shape of the modulation was found (see Figure 1, right panel) with a pulsed fraction of $\sim 11 \%$ (i.e. the semi amplitude of the modulation divided by the mean source count rate).

During the BeppoSAX observation, a type-I thermonuclear burst was detected at the time 51597 MJD - 15:29:55s (see below for details). The same procedure used for Chandra data was applied to 10s bin MECS/BeppoSAX light curve in the 3-5 keV energy band, with exclusion of the burst data. The best-fit period is $(1013 \pm 14) \mathrm{s}$ with uncertainties at $1 \sigma$ confidence level. Using this period value, we folded the light curves and a sinusoidal shape of the modulation was found (see figure 2) with a pulsed fraction of $\sim 4 \%$.

We have also searched for periodicity in the XMM-Newton and INTEGRAL X-ray light curves, but no periodic signals were detected. 
Table 2: Spectral analysis results. A $N_{H}$ fixed to the Galactic column density was included in the fit. Error are given at $90 \%$ confidence level for one parameter of interest $\left(\Delta \chi^{2}=2.71\right)$. The absorbed $1-10 \mathrm{keV}$ flux are reported in units of $10^{-11} \mathrm{erg} \mathrm{cm}^{-2} \mathrm{~s}^{-1}$. 1: BeppoSAX spectrum before the burst event; 2:BeppoSAX spectrum after the burst event; 3: XMM-Newton average spectrum; 4:Chandra average spectrum: 5: INTEGRAL average spectrum

\begin{tabular}{|c|c|c|c|c|c|c|c|c|}
\hline & $\begin{array}{c}\mathrm{kT}_{\mathrm{BB}} \\
k e V\end{array}$ & $\begin{array}{c}T_{0} \\
k e V\end{array}$ & $\begin{array}{l}\mathrm{kT}_{\mathrm{e}} \\
\mathrm{keV}\end{array}$ & $\tau$ & $n_{\mathrm{BB} 2}$ & $\begin{array}{c}n_{\text {COMPTT }} \\
10^{-3}\end{array}$ & $\begin{array}{c}\text { Flux } \\
10^{-11} \mathrm{erg} \mathrm{cm}^{-2} \mathrm{~s}^{-1}\end{array}$ & $\overline{\chi^{2} / \text { d.o.f }}$ \\
\hline 1 & $\ldots$ & $0.22 \pm 0.04$ & $7_{-3}^{+41}$ & $3.6_{-3.3}^{+2.6}$ & $\ldots$ & $8.6_{-8.5}^{+15.3}$ & 15.2 & $156 / 127$ \\
\hline 2 & $0.49 \pm 0.04$ & $0.18 \pm 0.02$ & $2.6 \pm 0.2$ & $6.8 \pm 0.6$ & $21 \pm 16$ & $38 \pm 4$ & 18.9 & $155 / 128$ \\
\hline 3 & $\ldots$ & $0.068 \pm 0.002$ & $15_{-10}^{+2}$ & $2_{-1}^{+9}$ & $\ldots$ & $86 \pm 20$ & 8.9 & $573 / 387$ \\
\hline 4 & $0.34 \pm 0.04$ & $0.13 \pm 0.05$ & $5_{-2}^{+24}$ & $6 \pm 2$ & $3.9_{-2.5}^{+2.7}$ & $0.7 \pm 0.4$ & 0.7 & $280 / 284$ \\
\hline 5 & $\ldots$ & $<1.1$ & $21_{-16}^{+32}$ & $<2.4$ & $\ldots$ & $<50$ & 11.0 & $3 / 7$ \\
\hline
\end{tabular}

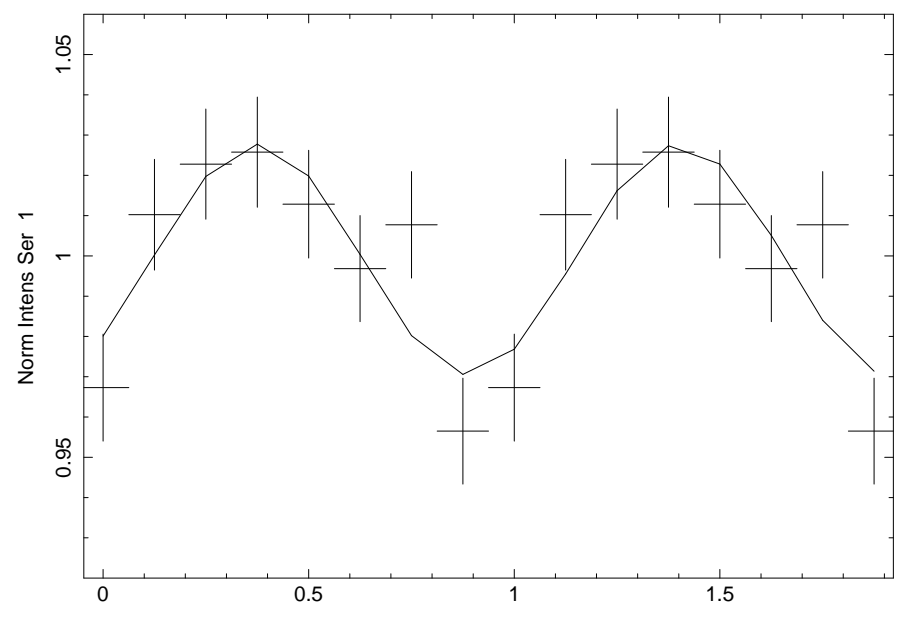

Figure 2: The efolding phase using MECS 3-5 keV data

In the soft X-ray band, two thermonuclear X-ray bursts have been detected, during the Chandra and BeppoSAX observations. The first HRC/Chandra burst event was described by Homer et al. (2001). For the second, not yet reported in the literature, we extracted the MECS/BeppoSAX light curve in the 1.5-10 keV keV energy band and we detected an X-ray burst starting at 51597 MJD (15:29:55 s), with a decay time of $\tau \sim 22 \mathrm{~s}$, computed from exponential fits to the burst decay profile. Fitting the spectrum (extracted at the burst peak with exposure time of $\sim 10 \mathrm{~s}$ ) with a simple blackbody model, a thermal temperature of $1.6 \pm 0.1 \mathrm{keV}$ with a extrapolated flux of $3.3 \times 10^{-9} \mathrm{erg} \mathrm{cm}^{-2} \mathrm{~s}^{-1}$ in 0.1-30 keV energy band has been derived. 


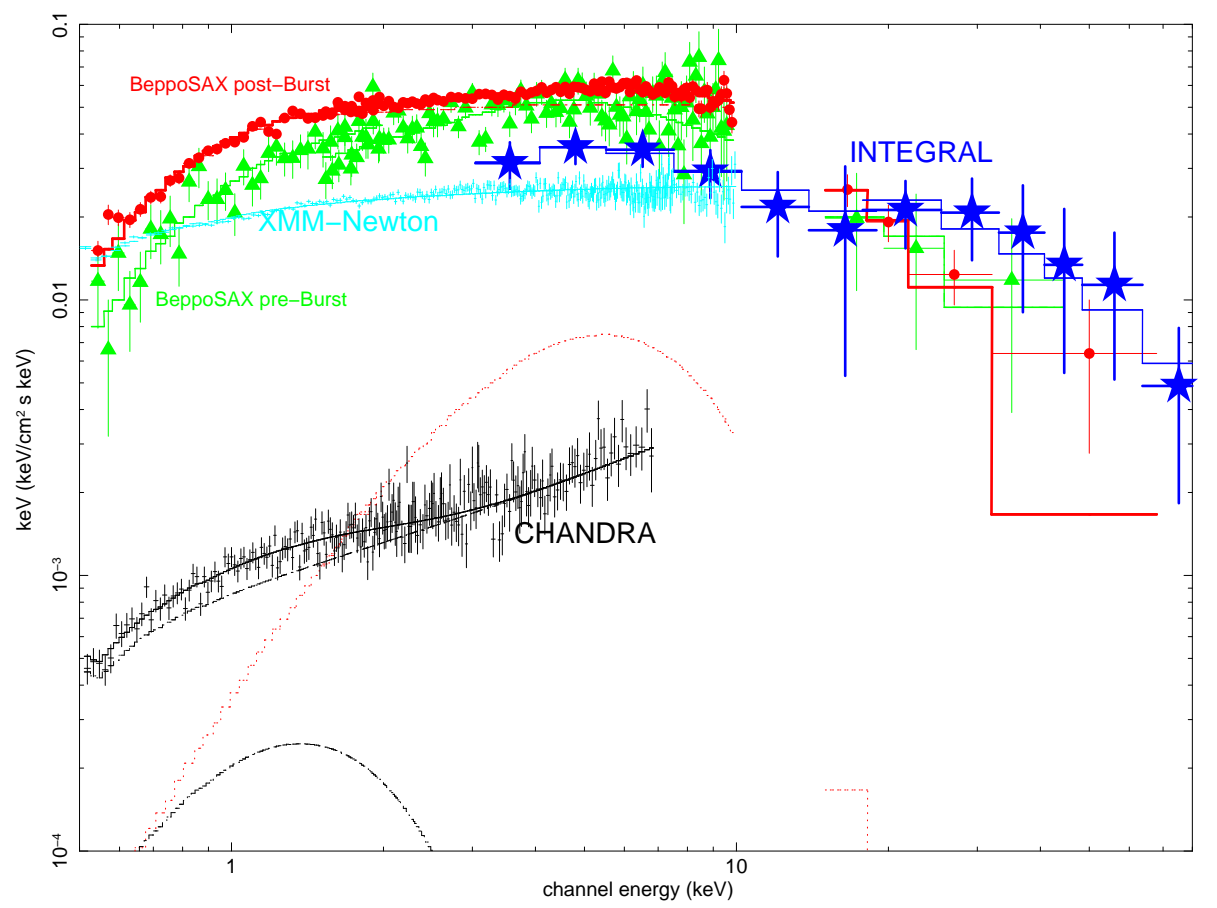

Figure 3: The BeppoSAX, Chandra, XMM-Newton and INTEGRAL spectra are shown together with the total model and its components.

\section{Spectral behavior of the persistent emission}

We extracted the BeppoSAX spectra before the burst event with the LECS, MECS and PDS exposure times of 1.6, 3.4 and $1.5 \mathrm{ks}$, and after the burst event with the LECS, MECS and PDS exposure times of $30.1,67.5$ and $31.2 \mathrm{ks}$, respectively.

IBIS and JEM-X spectra are produced summing up spectra of the source from each science windows in the period for which both instrument data are available and source was detected above $5 \sigma$. The exposure times are $103 \mathrm{ks}$ and $36 \mathrm{ks}$ for IBIS and JEM-X, respectively. For Chandra and XMM-Newton observations, to extract the spectra we use the total exposure time, 18.8 and $23.5 \mathrm{ks}$, respectively.

Each spectrum was fitted with a model of thermal Comptonization, in XSPEC by COMPTT (Titarchuk 1994; a spherical geometry was assumed), absorbed by a column density, $N_{\mathrm{H}}$. Adding a blackbody component (modeled in XSPEC by BBODYRAD model) resulted in a substantial fit improvement for Chandra and BeppoSAX (after the burst) data. This reduces $\chi^{2} /$ d.o.f. from $452 / 310$ to $374 / 308$ for the Chandra data and from 173/130 to $155 / 128$ for the BeppoSAX data, with the low corresponding F-test chance probabilities of $4 \times 10^{-13}$ and $9 \times 10^{-4}$, respectively.

The spectral fit results are reported in Table 2 and spectra are shown in Figure 3 in different colors.

\section{Discussion}

The binary system $4 \mathrm{U}$ 0513-40 in NGC 1851 exhibits a clear periodic signal with $P \simeq 17$ min 
in soft X-ray. This signal is sinusoidal and has an amplitude from $\sim 4 \%$ to $\sim 10 \%$. It is observed only in two observations (Chandra and BeppoSAX after the burst) when the source is in a typical high/soft state. This result is independent from the extrapolated luminosity of the system, which spans from 0.7 to $5.2 \times 10^{36} \mathrm{erg} \mathrm{s}^{-1}$ in the $0.5-50 \mathrm{keV}$ energy band, for Chandra and BeppoSAX (after the burst), respectively. This periodicity is not seen when the source is in a low/hard state (XMM-Newton and INTEGRAL data).

The far-ultraviolet photometry obtained with the Hubble Space Telescope has shown the same periodicity described here (Zurek et al. 2009). These timing properties seen in UV/optical observations and the eclipse observed in the X-ray band imply that the origin of this modulation is of orbital nature and the inclination angle is higher than $80^{\circ}$ (Arons and King 1993).

\section{Acknowledgments}

The authors acknowledge the ASI financial support via ASI-INAF contract I/033/10/0.

\section{References}

[1] Arons J. \& King I. R.,1993, ApJ, 413, L121

[2] Boella, G.; Butler, R. C.; Perola, G. C. et al., A\&AS, 122, 299

[3] Chiappetti L. \& Dal Fiume D., 1997, in Proc. Fifth Workshop, data Analysis in Astronomy, 101

[4] Courvoisier T. J.-L. et al., 2003, A\&A 411, L53

[5] Federici M., Martino B. L. and Natalucci L., 2009, PoS2009-092

[6] Fiocchi M. et al., 2008, A\&A, 492, 557

[7] Fiore F., Guainazzi M. \& Grandi P. 1999, Cookbook for BeppoSAX NFI Spectral Analysis Cookbook for BeppoSAX NFI Spectral Analysis (www.asdc.asi.it/bepposax/software/cookbook)

[8] Homer L. et al., 2001, ApJ, 550, L155

[9] in 't Zand J., Jonker P. \& Markwardt C., 2007, A\&A, 465, 953

[10] Israel G. \& Stella L., 1996, ApJ, 468, 369

[11] Lund N., et al. 2003, A\&A, 411, L231

[12] Maccarone T. et al. 2010, MNRAS, 406, 2087

[13] Nelemans G. \& Jonker P. G., 2006, astro, arXiv:astro-ph/0605722

[14] Turner M.J.L., Abbey A.F., Arnaud M. et al. 2001, A\&A 365, L27

[15] Titarchuk L., 1994, ApJ, 434, 570

[16] Ubertini P. et al., 2003, A\&A, 411, L131

[17] Weisskopf M. C., 1999, AAS, 195, 9601

[18] Winkler C. et al., 2003, A\&A, 411, 1

[19] Zurek D.R. et al., 2009, ApJ, 699, 1113 